\title{
Guaifenesin Has No Effect on Sputum Volume or Sputum Properties in Adolescents and Adults With Acute Respiratory Tract Infections
}

\author{
Agathe Hoffer-Schaefer, Henry J Rozycki MD, Melissa A Yopp, \\ and Bruce K Rubin MEngr MD MBA FAARC
}

\begin{abstract}
BACKGROUND: Guaifenesin (glyceryl guaiacolate ether [GGE]) has been studied as a cough suppressant and as an expectorant; however, published studies to date have failed to find a consistent benefit. METHODS: An 8-day multi-center clinical trial was conducted to study the effect of two 600-mg extended-release GGE tablets twice daily for 1 week on cold symptoms, sputum volume, and properties in adolescents and adults with productive cough from an acute respiratory tract infection (RTI). The study enrolled 378 subjects (GGE, $n=188$; and placebo, $n=190$ ) who were otherwise healthy and had an RTI for up to 5 days before enrollment. Subjects suffered from at least 2 of 3 symptoms of cough, thickened mucus, and chest congestion. A total of 151 GGE and 144 control subjects completed the full protocol. Single-sputum samples were collected from each subject on days $1,3,4$, and 8 of the study. The rheology and interfacial tension of sputum were measured, and 24-h collected samples from days 1 and 4 were analyzed for total volume and hydration. RESULTS: Symptoms in both the GGE and placebo groups improved to a similar degree over time. There were no significant differences between the GGE and placebo groups for sputum volume $(P=.41)$, percent solids $(P=.69)$, interfacial tension $(P=.88)$, elasticity $(P=.71)$, viscosity $(P=.45)$, or mechanical impedance $(P=.75)$. CONCLUSIONS: The recommended dose of GGE had no measurable effect on sputum volume or properties and is unlikely to be an expectorant or mucolytic when used to treat acute RTI. (ClinicalTrials.gov registration NCT01046136.) Key words: expectorant; mucus; guaifenesin; upper respiratory infection; cough therapy. [Respir Care 2014;59(5):631-636. (C) 2014 Daedalus Enterprises]
\end{abstract}

\section{Introduction}

Guaifenesin (3-(2-methoxyphenoxy)-1,2-propanediol), or glyceryl guaiacolate ether (GGE), was originally de-

\footnotetext{
All authors are affiliated with the Department of Pediatrics, Virginia Commonwealth University, Richmond, Virginia.

This study was funded by an investigator-initiated research grant from Reckitt Benckiser. The sponsor had full access to all data. The sponsor had no part in our data analysis or in writing the manuscript. The authors have disclosed no conflicts of interest.

Correspondence: Bruce K Rubin MEngr MD MBA FAARC, Department of Pediatrics, Virginia Commonwealth University School of Medicine, Richmond, VA 23298. E-mail: brubin@vcu.edu.
}

DOI: $10.4187 /$ respcare. 02640 rived from the guaiac tree. It has been used to treat respiratory disease since the 19th century. GGE is approved by the FDA as an over-the-counter expectorant, with sales of the Mucinex extended-release brand (Reckitt Benckiser Pharmaceuticals, Bristol, United Kingdom) accounting for approximately $\$ 135$ million in the United States annually. GGE has been studied both as a cough suppressant and as an expectorant; however, published studies have failed to

See the Related Editorial on Page 788

find a consistent benefit. ${ }^{1-5}$ The only available assessment of the effect of GGE in young adults with natural (ie, community-acquired) colds found no objective changes in sputum properties. ${ }^{6}$ 


\section{GuAIFENESIN AND UPPER RESPIRATORY TRACT INFECTIONS}

The purpose of this study was to determine whether two 600-mg extended-release GGE tablets (Mucinex) or matching placebo given twice daily for 7 days to subjects with an acute respiratory tract infection (RTI), that is a common cold, would improve the volume or properties of sputum and to evaluate the effect of GGE on subjective assessments of symptoms and symptom-associated quality of life. We are reporting only the objective measures of sputum volume and properties in this article.

\section{Methods}

\section{Subjects}

This was an 8-day multi-center double-blind randomized placebo-controlled trial comparing the administration of two 600-mg GGE tablets every $12 \mathrm{~h}$ for $7 \mathrm{~d}$ with placebo. Subjects were $12 \mathrm{y}$ of age or older and met the following inclusion criteria, as judged by their primary physician/investigator: (1) an RTI for $5 \mathrm{~d}$ or less; (2) a productive cough beginning within $72 \mathrm{~h}$ of the first dose of study medication; (3) at least 2 of 3 target symptoms of cough, thickened mucus, and chest congestion; (4) if female, a negative pregnancy test; and (5) likely to comply with the study requirements. Exclusion criteria included respiratory symptoms due to chronic pulmonary disease (eg, cystic fibrosis, COPD, asthma, allergic rhinitis), had been febrile $\left(>101^{\circ} \mathrm{F}\right)$ in the previous $7 \mathrm{~d}$, or clinically important physical or psychiatric disorders that could interfere with adherence or study outcomes. Subjects were also excluded if they were taking prescription or over-thecounter cough or cold medications. Concomitant nasal symptoms were not regarded as an exclusion criterion. Randomization of consenting eligible subjects was done by sequential number assignment, blocked by study site to achieve an approximately equal distribution for each group. Masking was achieved by packaging GGE and matching placebo product in identical blister packs and delivering them to each study site with no identifying markings other than the protocol and randomization numbers. Codes were withheld from patients and personnel until the study was completed and data locked.

Approval by the institutional review board at each study site was obtained before study initiation, and written informed consent was obtained from each subject before enrollment. Analysis of symptom scoring and quality-oflife data are not within the scope of this article. A portion of those results have been published. ${ }^{7}$

\section{Sputum Collection}

Spontaneously produced sputum was collected from each subject during morning clinic on days $1,3,4$, and 8 . On days 1,3 , and 4 , sputa were collected immediately before the test drug was administered and at 3 and $6 \mathrm{~h}$ after. On day 8 , only a baseline sample was collected. Sputum was

\section{QUICK LOOK}

\section{Current Knowledge}

Guaifenesin (GGE) is approved by the FDA as an overthe-counter expectorant, with sales accounting for approximately $\$ 135$ million in the United States annually. GGE has been studied both as a cough suppressant and as an expectorant with inconsistent findings.

\section{What this paper contributes to our knowledge}

At the recommended dose, GGE has no measurable effect on sputum volume or sputum properties and is unlikely to be an expectorant or mucolytic when used to treat acute respiratory infection.

visually separated from saliva and detritus, and samples were stored at $-80^{\circ} \mathrm{C}$ until analysis. Twenty-four-hour collections of sputa were done by subjects at home on days $1,2,4$, and 5; stored in a sputum cup in home refrigerators; and returned to the clinic on days 2 and 8 , whereby they were frozen at $-80^{\circ} \mathrm{C}$. Sputa were thawed on ice for analysis. Analysis of sputum biophysical properties was performed immediately upon thawing.

Sputum samples were analyzed only if they met visual examination criteria for sample integrity. Samples that were clearly watery and degraded or contaminated by saliva were not analyzed. Ideal samples were also free of food or other detritus. Samples that were not identifiable because of missing or illegible labels were discarded without analysis. The median number of sputum samples provided by

Table 1. Sample Distribution

\begin{tabular}{|c|c|c|c|}
\hline & $\begin{array}{c}\text { GGE } \\
(n=188)\end{array}$ & $\begin{array}{l}\text { Placebo } \\
(n=190)\end{array}$ & $\begin{array}{c}\text { Total } \\
(n=378)\end{array}$ \\
\hline \multicolumn{4}{|l|}{ Day 1} \\
\hline Predrug & 118 & 117 & 235 \\
\hline $3 \mathrm{~h}$ & 51 & 62 & 113 \\
\hline $6 \mathrm{~h}$ & 107 & 109 & 216 \\
\hline \multicolumn{4}{|l|}{ Day 3} \\
\hline Predrug & 99 & 95 & 194 \\
\hline $3 \mathrm{~h}$ & 55 & 62 & 117 \\
\hline $6 \mathrm{~h}$ & 85 & 82 & 167 \\
\hline \multicolumn{4}{|l|}{ Day 4} \\
\hline Predrug & 106 & 110 & 216 \\
\hline $3 \mathrm{~h}$ & 44 & 43 & 87 \\
\hline $6 \mathrm{~h}$ & 84 & 86 & 170 \\
\hline Day 8 & 40 & 47 & 87 \\
\hline \multicolumn{4}{|c|}{$\begin{array}{l}\text { Number of sputum samples available for analysis for each scheduled collection by group } \\
\text { assignment } \\
\text { GGE = guaifenesin }\end{array}$} \\
\hline
\end{tabular}


each subject was 6 (interquartile range 4-7). The sample distribution per collection is outlined in Table 1 .

\section{Rheology}

To assess whether GGE improves the properties of sputum that influence the effectiveness of sputum clearance, each of the serially collected sputa was evaluated for dynamic rheology using a rheometer (AR1500ex, TA Instruments, New Castle, Delaware). Parallel plate geometry was used across the dynamic frequency range of stressstrain of a $20-\mu \mathrm{L}$ sputum sample over driving frequencies $0.01-1000 \mathrm{rad} / \mathrm{s}$ using nondestructive creep transformation and triplicate analyses. Rheology measurements were made if the measured strain from 1 to $100 \mathrm{rad} / \mathrm{s}$ was linear and reproducible. ${ }^{8}$ By a priori determination, specimens with nonreproducible results indicated by a $>20 \%$ variability in the linear testing range were considered degraded or contaminated with air, and results were not recorded.

\section{Interfacial Tension}

Interfacial tension was evaluated using the du Noüy ring distraction method. A $90 \%$ platinum $/ 10 \%$ iridium ring was pulled from the mucus at a distraction velocity of $10 \mathrm{~mm} / \mathrm{s}$ until separation was achieved. Interfacial tension was measured at the air-mucus interface by a strain gauge connected to the ring (Tensiomat Model 21, Fisher Scientific, Pittsburgh, Pennsylvania). A purpose-made calibrated ring with a circumference of $1.7145 \pm 0.0381 \mathrm{~cm}$ was used as described previously. ${ }^{9}$ Interfacial tension measures the force separating a gel and gas phase; this is called surface tension when measured at the interface between a liquid and air. The work of adhesion of a sessile drop can be calculated by Young's equation as work of adhesion $=\gamma(1+\cos \theta)$, where $\gamma$ is the interfacial tension of mucus in air and $\theta$ is the contact angle of mucus on the epithelium. Sputum with high work of adhesion is more difficult to clear by cough.

\section{Mucus Hydration and Volume}

To determine whether GGE works as an expectorant and increases the volume of mucus or fluid produced, the total weight and hydration (\% solid material) of the two 24-h samples were calculated by measuring the wet weight of the sputum, freeze-drying the sputum, and determining the ratio of the solid weight to wet weight to obtain the percent solids. ${ }^{10}$

\section{Statistical Analysis}

Prestudy sample size determination was based on previous studies of sputum properties, so 120 subjects per group were needed to detect a 50\% change in sputum dynamic viscosity
Table 2. Subject Demographics

\begin{tabular}{lccc}
\hline \hline & $\begin{array}{c}\text { GGE } \\
(n=188)\end{array}$ & $\begin{array}{c}\text { Placebo } \\
(n=190)\end{array}$ & $P$ \\
\hline Age (mean \pm SD y) & $41.2 \pm 13.9$ & $40.8 \pm 15.0$ & .41 \\
Sex (\%) & & & \\
$\quad$ Male & 49 & 56 & .22 \\
$\quad$ Female & 51 & 44 & \\
Ethnicity (\%) & & & \\
Hispanic/Latino & 7 & 5 & .41 \\
Not Hispanic/Latino & 93 & 95 & \\
Race (\%) & & & \\
$\quad$ Caucasian & 61 & 61 & .34 \\
African-American & 37 & 33 & \\
Asian & 2 & 4 & \\
American Indian/Alaska Native & 1 & 2 & \\
$\quad$ Native Hawaiian/Pacific Islander & 1 & 0 &
\end{tabular}

Subject demographics for the placebo and guaifenesin groups. There were no statistically significant differences between groups by chi-square analysis or $t$ test for age. $\mathrm{GGE}=$ guaifenesin

with $80 \%$ power. The goal of the study was to enroll at least 375 subjects and collect data on 300 to provide a sufficient sample to have an $80 \%$ power to detect a statistically significant $(P<.05)$ difference between groups for both rheology and sputum volume outcome measures. Although we were unable to collect uncontaminated sputum from all participants at every time point, post hoc power calculation, using the means and variability of the results obtained, confirmed that the study sample size was sufficient to detect any clinically important changes in the volume or biophysical properties of the sputum. ${ }^{11}$

Data were logged in spreadsheets and analyzed by JMP 9.0 or StatView 5.0 (both from SAS Institute, Cary, North Carolina). Analyses were based on per protocol subjects defined as those who met all inclusion and exclusion criteria; were $100 \%$ dosing-compliant from days 1 to 4 ; had $80 \%$ of their diaries complete on days 1 and 4, with some diary information on days 2 and 3; and had their visits on the designated study day. Subject demographics and sputum were compared among groups using the $t$ test for continuous variables, MannWhitney $\mathrm{U}$ test for categorical variables, and chi-square test for proportions. Sputum rheology and interfacial tension were analyzed by two-way analysis of variance (ANOVA). Post hoc correction for multiple comparisons was calculated using the Sidak method. Where applicable, results are reported as means \pm standard error of the mean. Significance was defined as $P<.05$.

\section{Results}

\section{Patient Demographics}

The study enrolled 378 subjects (GGE, $n=188$; and placebo, $n=190)$ at 12 centers; the subjects were other- 


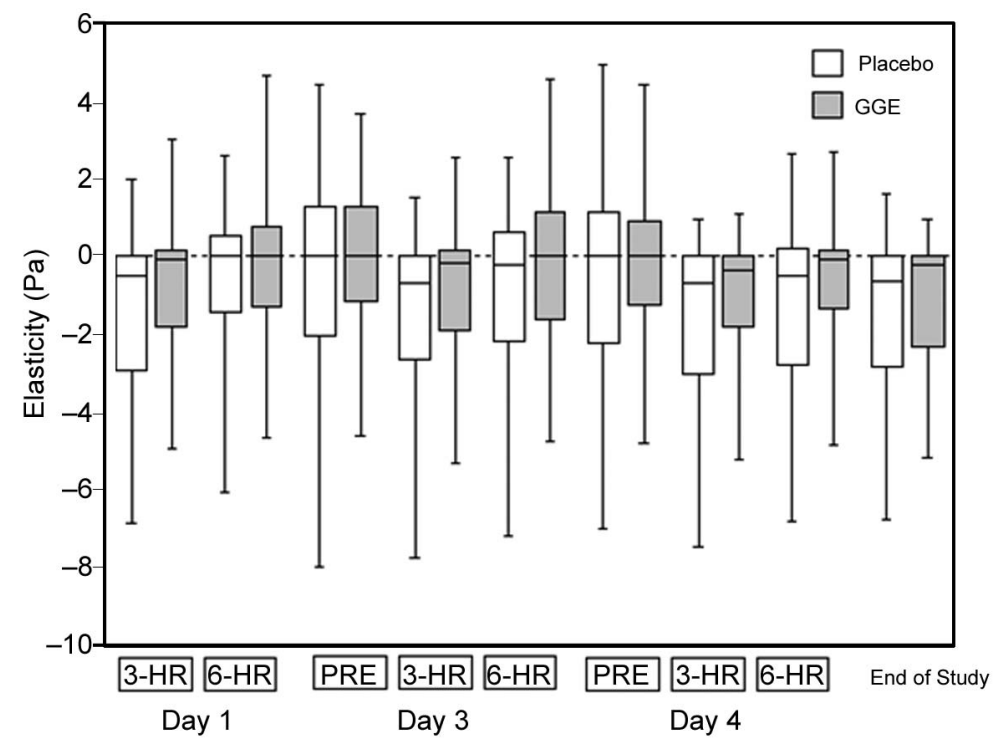

Fig. 1. Changes in elasticity from baseline in the placebo and GGE (glyceryl guaiacolate) groups over the course of the study. There were no significant differences in the change from baseline between the two groups by ANOVA. Whiskers represent $95 \% \mathrm{Cl}$.

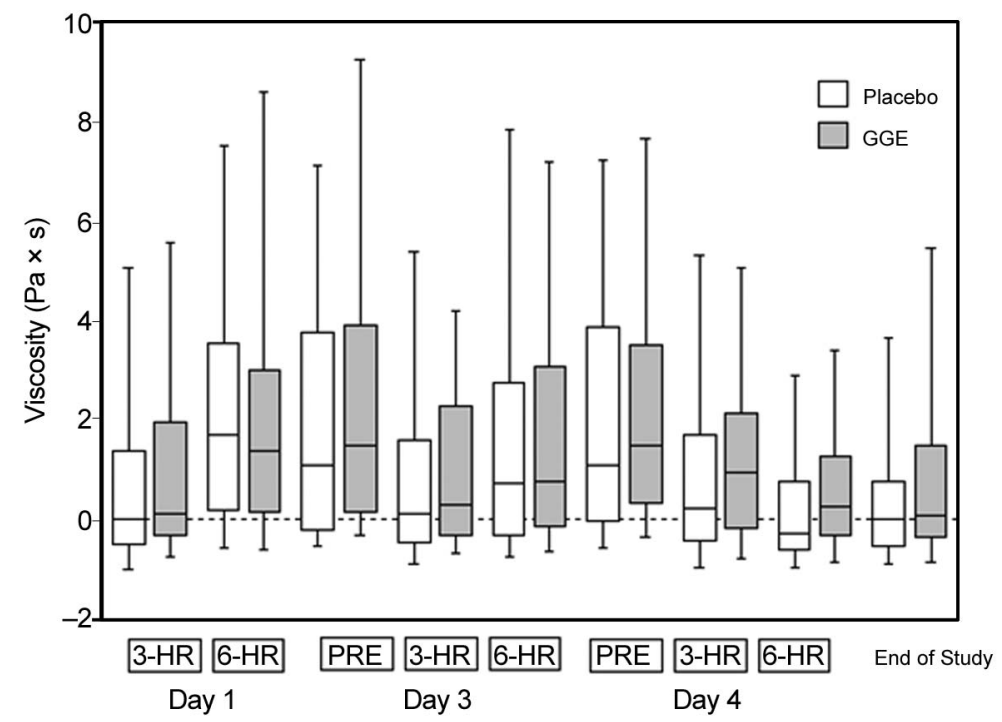

Fig. 2. Changes in viscosity from baseline in the placebo and GGE (glyceryl guaiacolate) groups over the course of the study. There were no significant differences in the change from baseline between the two groups by ANOVA. Whiskers represent $95 \% \mathrm{Cl}$.

wise healthy and had symptomatic RTI for up to $5 \mathrm{~d}$ before enrollment. There were 295 subjects (151 GGE and 144 control) who completed the full protocol. There were no significant differences between groups for demographics (Table 2).

\section{Rheology}

As determined by two-way ANOVA, there were no significant differences with respect to changes from baseline in the study between the GGE and placebo subjects for elasticity $\left(\mathrm{G}^{\prime}, P=.71\right)$ (Fig. 1), viscosity $\left(\mathrm{G}^{\prime \prime}, P=.45\right.$ (Fig. 2), and mechanical impedance ( $\left.\mathrm{G}^{*}, P=.75\right)$.

\section{Interfacial Tension}

There were no significant treatment differences between groups concerning interfacial tension at any time point $(P=.88)$ (Fig. 3).

\section{Mucus Hydration and Volume}

There were no significant differences in the wet weight between groups at the first collection (GGE, $4.70 \pm 0.471 \mathrm{~g}$; 


\section{GuAIFENESIN AND UPPER RESPIRATORY TRACT INFECTIONS}

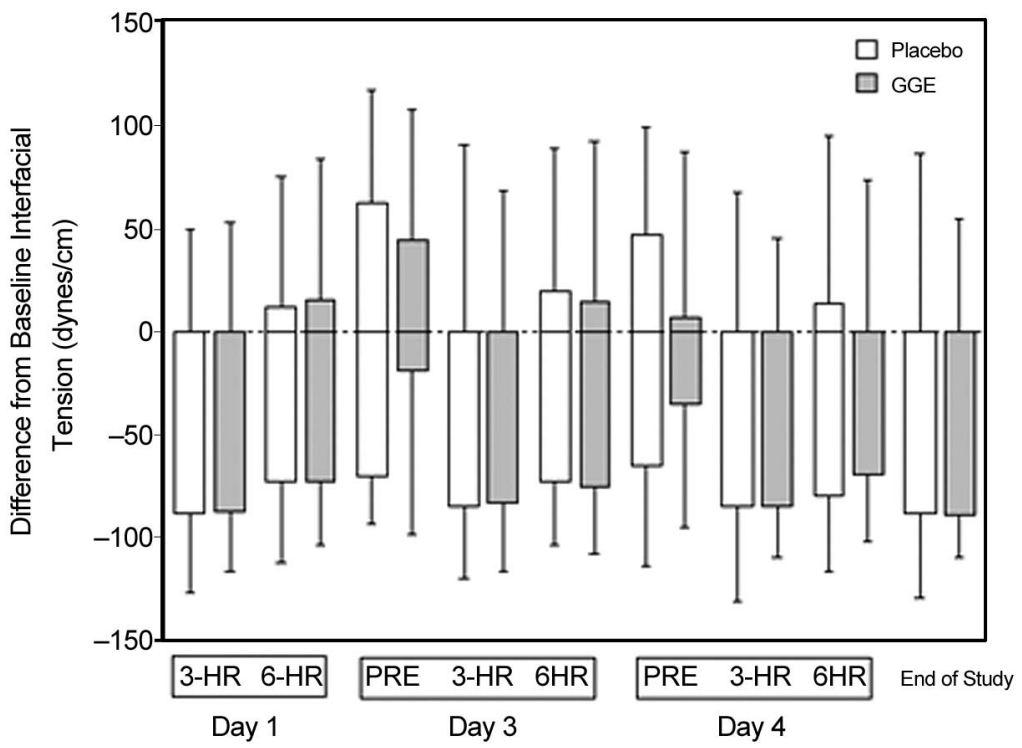

Fig. 3. Changes in interfacial tension from baseline in the placebo and GGE (glyceryl guaiacolate) groups over the course of the study. There were no significant differences in the change from baseline between the two groups by ANOVA. Whiskers represent $95 \% \mathrm{Cl}$.

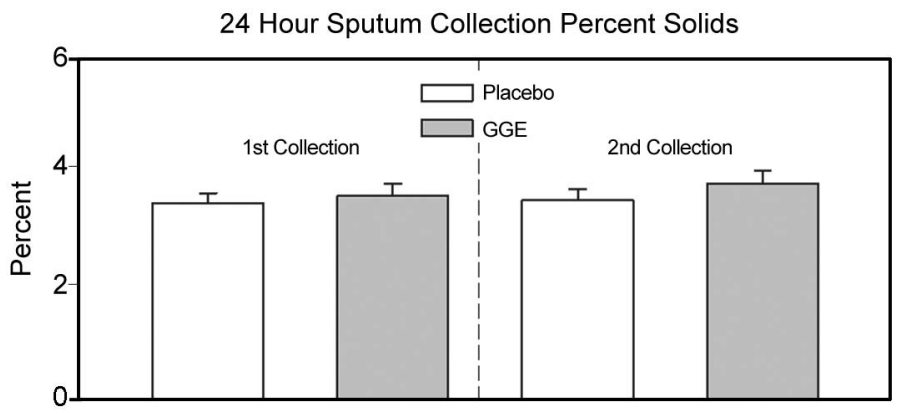

Fig. 4. Sputum percent solids for the first collection (days 1 and 2) and the second collection (days 4 and 5) in the control and GGE (glyceryl guaiacolate) groups. By $t$ test, there were no significant differences between the two groups in either the first collection $(P=.69)$ or the second collection $(P=.33)$.

placebo, $6.083 \pm 0.754 \mathrm{~g} ; P=.12$ ) or second collection (GGE, $4.292 \pm 0.460 \mathrm{~g}$; placebo, $4.718 \pm 0.518 \mathrm{~g} ; P=.54$ ) or in the change between the two collections (GGE, $-0.981 \pm 0.593 \mathrm{~g}$; placebo, $-2.081 \pm 0.821 \mathrm{~g} ; P=.14$ ). Similarly, there were no significant differences in the percent solids between the groups (Fig. 4) in the two collections or in the change between the collections (GGE, $0.191 \pm 0.223 \%$; placebo, $0.042 \pm 0.236 \% ; P=.65)$.

\section{Discussion}

In this large randomized clinical trial, there were no consistent statistically significant changes in sputum properties comparing placebo with GGE for therapy of acute symptoms of RTI in subjects without underlying chronic pulmonary disease. The absence of changes in sputum rheology and interfacial tension, independent of withingroup symptom decrease over time, makes it unlikely that
GGE is a mucolytic or that GGE therapy would improve mucociliary or cough clearance. Similarly, the lack of difference in sputum wet volume or dry weight makes it unlikely that GGE has significant expectorant properties in healthy subjects with an acute upper RTI. Although the upper respiratory quality of life improved over the course of the study in both the GGE and placebo groups, this improvement was not accompanied by changes in sputum properties.

We conclude that extended-release GGE (Mucinex) administered at the recommended dose is no more effective than a placebo in changing sputum properties. Although it is possible that the negative results could be due to an inadequate dose, the drug dosage studied here is consistent with current dosing recommendations for GGE as an overthe-counter medication to ease cold symptoms. It is also possible that the drop off in sample number over the course of the study could confound the results because those sub- 


\section{GuAIFENESIN AND UPPER RESPIRATORY TRACT INFECTIONS}

jects with improvement in their productive cough or sputum production may have been omitted from the sampling. Another possible limitation is that the diagnosis of RTI was made clinically and not confirmed by microbiological testing. However, the clinical assessment was performed by experienced clinicians, and the clinical conditions matched those for which GGE is most commonly used.

It has been suggested that GGE is either an expectorant or a mucolytic (requiring very different mechanisms of action) and that changes in sputum properties are responsible for clinical effectiveness. If GGE were an expectorant, ${ }^{4,12,13}$ we would expect to see increased sputum volume and hydration. This was not supported by the results of sputum volume or percent solid measurements in our study. If GGE were a mucolytic, ${ }^{14,15}$ we would expect to see decreased viscoelasticity. In our study, we saw no evidence of either expectorant or mucolytic properties either for GGE or for the placebo. Furthermore, there were no changes in sputum properties that would be expected to improve mucociliary or cough clearance. In contrast to an earlier in vitro study showing that GGE decreased mucociliary transport when applied directly to a ciliated epithelium, ${ }^{14}$ a more recent study showed a dose-dependent increase in mucus transport when GGE was introduced into airway cell culture. ${ }^{15}$ These data from a clinical study in subjects with an acute upper RTI clearly show no evidence of mucolytic properties and no temporal or drug-associated changes in viscoelasticity or sputum volume.

The results of this study, which focused on the properties of mucus during RTIs, are consistent with published studies showing that GGE is not an effective medication for treating acute RTIs. ${ }^{1-5}$ The placebo effect has been reported to be as high as $85 \%$ in studies of cough therapy, ${ }^{16}$ and given the absence of biophysical findings, this may be the best explanation for any perceived benefits for GGE.

\section{REFERENCES}

1. U.S. Food and Drug Administration. Cold, cough, allergy, bronchodilator, and antiasthmatic drug products for over-the-counter human use: tentative final monograph for over-the-counter anticholinergic drug products and expectorant drug products. Fed Reg 1982;47: 30002 .

2. Kuhn JJ, Hendley JO, Adams KF, Clark JW, Gwaltney JM. Antitussive effect of guaifenesin in young adults with natural colds. Chest 1982;82(6):713-718.

3. Bolser DC. Cough suppressant and pharmacologic protussive therapy: ACCP evidence-based clinical practice guidelines. Chest 2006; 129(1 Suppl):238S-249S.

4. Smith SM, Schroeder K, Fahey T. Over-the-counter (OTC) medications for acute cough in children and adults in ambulatory settings. Cochrane Database Syst Rev 2012;(1):CD001831.

5. Chodosh S, Segal M. Current concepts: chronic bronchitis. N Engl J Med 1964;270(20):1057-1059.

6. U.S. Food and Drug Administration. Draft guidance on marketed unapproved drugs: compliance policy guide: availability. Fed Reg 2003;68:60,702,703.

7. Albrecht A, Vernon M, Solomon G. Patient-reported outcomes to assess the efficacy of extended-release guaifenesin for the treatment of acute respiratory tract infection symptoms. Respir Res 2012;13: 118.

8. King M, Rubin BK. Mucus rheology, relationship with transport. In: Takishima T, editor. Airway secretion: physiological bases for the control of mucus hypersecretion. New York: Marcel Dekker; 1994: 283-314.

9. Albers GM, Tomkiewicz RP, May MK, Ramirez OE, Rubin BK. Ring distraction technique for measuring surface tension of sputum: relationship to sputum clearability. J Appl Physiol 1996;81(6):26902695.

10. Rubin BK, Ramirez O, King M. Mucus rheology and transport in neonatal respiratory distress syndrome and the effect of surfactant therapy. Chest 1992;101(4):1080-1085.

11. Rubin BK. Designing clinical trials to evaluate mucus clearance therapy. Respir Care 2007;52(10):1348-1361.

12. Chodosh S, Medici TC, Ishikawa S, Enslein K. Objective sputum changes associated with glyceryl guaiacolate in chronic bronchial diseases. Bull Physiopathol Respir 1973;9(2):452-456.

13. Rubin BK. Mucolytics, expectorants, and mucokinetic medications. Respir Care 2007;52(7):859-865.

14. Rubin BK. An in vitro comparison of the mucoactive properties of guaifenesin, iodinated glycerol, surfactant, and albuterol. Chest 1999; 116(1):195-200.

15. Seagrave J, Albrecht H, Park YS, Rubin B, Solomon G, Kim KC. Effect of guaifenesin on mucin production, rheology, and mucociliary transport in differentiated human airway epithelial cells. Exp Lung Res 2011;37(10):606-614.

16. Eccles R. The powerful placebo in cough studies? Pulm Pharmacol Ther 2002;15(3):303-308.

This article is approved for Continuing Respiratory Care Education credit. For information and to obtain your CRCE

(free to AARC members) visit

www.rcjournal.com

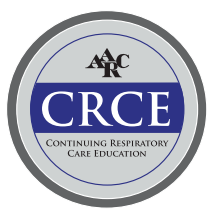

